\title{
TaqMan probes as blocking agents for enriched PCR amplification and DNA melting analysis of mutant genes
}

\author{
Irina V. Botezatu, Irina O. Panchuk, Anna M. Stroganova, Anastasia I. Senderovich, Valentina N. \\ Kondratova, Valery P. Shelepov, and Anatoly V. Lichtenstein \\ N.N. Blokhin Cancer Research Center, Moscow, Russia
}

BioTechniques 62:62-68 (February 2017) doi 10.2144/000114515

Keywords: mutation detection; DNA melting analysis; TaqMan probes; blocking agents; NRAS; BRAF

Supplementary material for this article is available at www.BioTechniques.com/article/114515.

Asymmetric PCR and DNA melting analysis with TaqMan probes applied for mutation detection is effectively used in clinical diagnostics. The method is simple, cost-effective, and carried out in a closed-tube format, minimizing time, labor, and risk of sample cross-contamination. Although DNA melting analysis is more sensitive than Sanger sequencing (mutation detection thresholds are $~ 5 \%$ and 15\%-20\%, respectively), it is less sensitive than more labor-intensive and expensive techniques such as pyrosequencing and droplet digital PCR. Here, we demonstrate that, under specially selected conditions of asymmetric PCR, TaqMan probes can play the role of blocking agents. Preferential blocking of the wild-type allele brings about enriched amplification of mutant alleles. As a result, an $\sim 10$-fold increase in the detection sensitivity for mutant BRAF and NRAS genes was achieved.

Identification of gene mutations in tumor DNA is an important step in the initial examination of cancer patients. With the increased availability of tumor genome sequences, the role of clinical gene diagnostics is growing. Basic research has defined driver mutations specific to particular types of tumor (for example, KRAS, NRAS, and BRAF mutations in melanoma, colon cancer and lung cancer) (1-3). Translated into clinical practice, these findings facilitate differential diagnosis and determination of disease prognosis, as well as identification of the drug resistance of the tumors and, hence, therapy selection (4). However, clones of mutant, drug resistant cells are often very small in number and, therefore, difficult to detect in tumors $(5,6)$. To overcome this problem, highly sensitive mutation detection methods are needed.

Sanger sequencing (the gold standard of clinical diagnostics) lacks sensitivity $(7,8)$ and gives an unacceptably high rate of false- negative results. So, the development of simple, high-throughput, and highly sensitive mutation scanning techniques for clinical genetic testing is particularly important. High-resolution melting analysis (HRMA) (9-12) and its variant, DNA melting analysis (DMA) using TaqMan probes (13-15), meet these criteria to a large extent. TaqMan probes were initially intended for monitoring real-time PCR (16). The rationale of DMA is that a singlestranded amplicon synthesized in the first stage (asymmetric PCR) is hybridized in the second stage (DMA) with a TaqMan probe, and the duplex melting curve is analyzed to detect the presence of a mutation.

The DMA method is simple in application, cost-effective, and is carried out in the closed-tube format, which minimizes time, labor, and sample cross-contamination. It is more sensitive than Sanger sequencing (limit of detection is 5\% mutant alleles), yet it is surpassed in this respect by more expensive and laborintensive techniques, such as pyrosequencing $(7,8)$ and droplet digital PCR (Application note. Yang et al., 2015. Droplet Digital PCR: Multiplex Detection of KRAS Mutations in Formalin-Fixed, Paraffin-Embedded Colorectal Cancer Samples. BioTechniques 58:205-206.).

To enhance the sensitivity of DMA, which otherwise appeared to be optimal for clinical genetic testing, we took advantage of oligonucleotide probes to reduce the extension rates of Taq polymerase (17). This makes possible the enriched amplification of a mutant allele because a TaqMan probe/template duplex containing a mismatch is less stable (and therefore less obstructive for the enzyme) than a probe/template duplex containing the wild-type sequence. As a result, we have developed a protocol for asymmetric PCR-DMA, which demonstrated an approximately 10-fold higher sensitivity in mutation scanning of the $B R A F$ and NRAS genes.

\section{METHOD SUMMARY}

For mutation detection, we use TaqMan probes to block amplification of the wild-type allele during asymmetric PCR in order to enrich the mutant allele. This modification increases mutation detection sensitivity by DNA melting analysis $\sim 10$-fold while using the same TaqMan probes. 


\section{Materials and methods}

DNA samples

Tumor tissue samples (lung cancer, colorectal cancer, and melanoma) were obtained from the clinic of the N.N. Blokhin Cancer Research Center (Moscow, Russia).

DNA was isolated from tissues and cultured cells by deproteinization with phenol and chloroform. DNA from formalin-fixed paraffin-embedded (FFPE) tissues was isolated using a QIAamp DNA FFPE Tissue Kit (Qiagen, Valencia, CA) as recommended by the manufacturer. Concentrations of DNA were determined spectrophotometrically (Nano-Drop 1000, Thermo Fisher Scientific, Wilmington, DE).

For serial dilutions of mutant DNA with wild-type DNA, we used DNA from the human colorectal carcinoma cell line RKO [CRL 2577; American Type Culture Collection (ATCC), Manassas, VA] with a heterozygous mutation in the BRAF gene (V600E, GTG $\rightarrow$ GAG) and the human fibrosarcoma cell line HT1080 (CCL-121, ATCC) with a heterozygous NRAS mutation (Q61K, CAA $\rightarrow$ AAA).

\section{Sanger sequencing}

BRAF and NRAS amplicons were sequenced in both directions (Syntol, Moscow, Russia). DNA sequencing was done by means of a panel of BrightDye Terminator reagents (NimaGen B.V., Nijmegen, The Netherlands) with subsequent analysis of reaction products on an automatic ABI Prism 3130xI Genetic Analyzer (Thermo Fisher Scientific).

Thermodynamic DNA melting point predictions

The $T_{m}$ values of primers and probes was determined using the MeltCalc software (18).
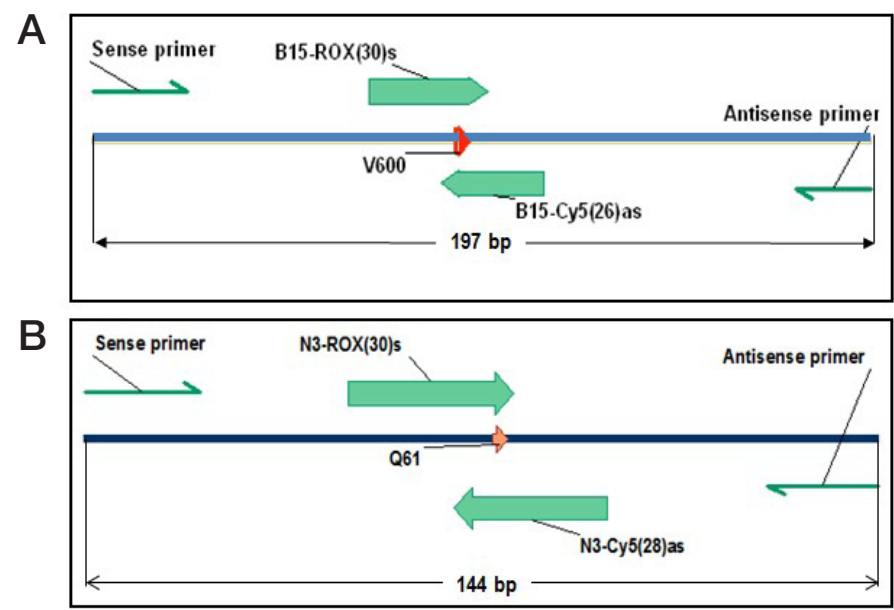

Figure1. Schematic diagram of the B15 and N3 amplicons. The amplicon name is derived from the gene name and exon number. The arrows show the locations of primers, TaqMan probes, and mutation hotspots. (The TaqMan probe name includes the amplicon name, fluorophore, oligonucleotide length, and sense or antisense direction.) (A) amplicon B15; (B) amplicon N3.

\section{Symmetric PCR}

Amplicons of the BRAF and NRAS genes (GenBank Accession no.s NC_000007.14 and NC_000001, respectively) as well as sequences of the primers and TaqMan probes are shown in Table 1. The primer and probe sequences were selected using the program Vector NTI Advance 10 (Thermo Fisher Scientific). Figure 1 illustrates the BRAF and NRAS amplicons along with the primers, TaqMan probes, and mutation hotspots (codons V600 and Q61, respectively). Sense and antisense TaqMan probes are shifted relative to each other to prevent their full-length hybridization when used simultaneously in PCR and DMA.

PCR was carried out in 96-well plates in a CFX96 (Bio-Rad Laboratories, Hercules, $\mathrm{CA}$ ) detection system. The reaction mixture $(25 \mu \mathrm{L})$ contained $50 \mathrm{mM}$ Tris- $\mathrm{HCl}, \mathrm{pH}$ 8.8; 50 mM KCl; 0.01\% Tween-20; 3 $\mathrm{mM} \mathrm{MgCl}_{2} ; 250 \mu \mathrm{M}$ each of dNTPs; 0.2x EvaGreen (Biotium, Hayward, CA); 1.25 U Hot-Rescue Taq polymerase (Syntol); 0.15 $\mu \mathrm{M}$ forward and reversed primers (Syntol); 1 MM N3-ROX(30) STaqMan probe (Syntol);

Table 1. Primers and TaqMan probes for asymmetric PCR and DNA melting analysis (DMA)

\begin{tabular}{|c|c|c|}
\hline $\begin{array}{l}\text { Genes, amplicons } \\
\text { (exon, codons, size) }\end{array}$ & $\begin{array}{l}\text { Primers (forward, reverse) } \\
\text { probes (sense, antisense) }\end{array}$ & Sequences, sizes \\
\hline \multicolumn{3}{|l|}{ BRAF } \\
\hline \multirow[t]{4}{*}{ B15 (exon 15 , c. $600-601,197$ bp) } & Primer, forward & 5 '-gagatctactgttttcctttactt ( 24 bases) \\
\hline & Primer, reverse & 5'-ggccaaaaatttaatcagt (19 bases) \\
\hline & Probe B15-ROX(30) & 5'-ROX-aggtgatttggtctagctacagtgaaatc-BHQ2 ( 30 bases) \\
\hline & Probe B15-Cy5(26) & 5'-Cy5-acccactccatcgagatttcactgta-BHQ2 (26 bases) \\
\hline \multicolumn{3}{|l|}{ NRAS } \\
\hline \multirow[t]{4}{*}{ N3 (exon 3, c. 61,144 bp) } & Primer, forward & 5'-cccaggattcttacagaaaac (21 bases) \\
\hline & Primer, reverse & 5 'tggcaaatacacagaggaag ( 20 bases) \\
\hline & Probe N3-ROX(30) & 5'-ROX-tgttggacatactggatacagctggacaag-BHQ2 (30 bases) \\
\hline & Probe N3-Cy5(28) as & 5'-Cy5- catggcactgtactcttcttgtccagct-BHQ2 (28 bases) \\
\hline
\end{tabular}

and $5 \mu \mathrm{L}$ DNA solution. PCR conditions were as follows: initial denaturation for 5 min at $95^{\circ} \mathrm{C}$, then $50-100$ cycles of $95^{\circ} \mathrm{C}$ for $13 \mathrm{~s}$ and $54^{\circ} \mathrm{C}$ for $2 \mathrm{~s}$ with fluorescence acquisition at $54^{\circ} \mathrm{C}$.

\section{Asymmetric PCR and DMA}

Asymmetric PCR was carried out to allow the excess synthesis of either the sense or antisense amplicon strands, thus allowing us to scan for mutations in either of the two strands. Selection of which of the two strands should be a target for the TaqMan probe can significantly affect discrimination between the wild-type and mutant alleles (Supplementary Figure S1). Thus, the ability to detect mutations in both strands of the amplicon can increase the reliability and sensitivity of the assay (14, 15).

Standard DMA conditions $\left(D M A_{s t}\right)$ $(14,15)$ : Asymmetric PCR was carried out in 96-well plates in a CFX96 (Bio-Rad Laboratories, Hercules, CA) detection system. The reaction mixture $(25 \mu \mathrm{L})$ contained $50 \mathrm{mM}$ Tris- $\mathrm{HCl}, \mathrm{pH}$ 8.8; 50 mM KCl; 0.01\% Tween-20; 3 mM $\mathrm{MgCl}_{2} ; 250 \mu \mathrm{M}$ each of dNTPs; 0.2x EvaGreen (Biotium); 1.25 U Hot-Rescue Taq polymerase (Syntol); and $5 \mu \mathrm{L}$ DNA solution. Depending on whether an excess of the sense or antisense amplicon strand had to be produced and tested, sense/ antisense (s/as) primer ratios were $10: 1$ or

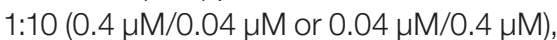
and $0.2 \mu \mathrm{M}$ TaqMan probe (antisense or sense, respectively) was added. In experiments aimed at revealing the inhibitory effect of the TaqMan probes on amplification efficiency, the control samples did not contain the TaqMan probes during PCR but were added to the reaction mixture just prior to DMA. PCR conditions 
were as follows: initial denaturation for 5 min at $95^{\circ} \mathrm{C}$, then 55 cycles of $13 \mathrm{~s}$ at $95^{\circ} \mathrm{C}$, $15 \mathrm{~s}$ at $57^{\circ} \mathrm{C}$ and $20 \mathrm{~s}$ at $72^{\circ} \mathrm{C}$ (fluorescence acquisition was at $72^{\circ} \mathrm{C}$ ). After PCR, the DNA was heated at $95^{\circ} \mathrm{C}$ for $1 \mathrm{~min}$, incubated at $55^{\circ} \mathrm{C}$ for $2 \mathrm{~min}$, after which it was melted from $55^{\circ} \mathrm{C}$ to $85^{\circ} \mathrm{C}$ (increments of $0.4^{\circ} \mathrm{C}$, dwell time of $6 \mathrm{~s}$, rate of heating of $3.3^{\circ} \mathrm{C} / \mathrm{s}$ ).

Modified DMA conditions (DMA ${ }_{\text {mod }}$ ): Asymmetric PCR was carried out in 96-well plates in a CFX96 detection system. The incubation mixture $(25 \mu \mathrm{L})$ contained 50 $\mathrm{mM}$ Tris- $\mathrm{HCl}, \mathrm{pH} 8.8,50 \mathrm{mM} \mathrm{KCl,} \mathrm{0.01 \%}$ Tween-20, $3 \mathrm{mM} \mathrm{MgCl}$, $250 \mu \mathrm{M}$ each of the dNTPs, 0.2× EvaGreen (Biotium), sense and antisense primers with s/as ratios of $15: 1$ or $1: 15(0.4 \mu \mathrm{M}$ of one and $0.027 \mu \mathrm{M}$ of the other), both sense and antisense TaqMan probes (0.25 $\mu$ M each), 0.3 or 0.5 U Hot-Rescue Taq polymerase (for N3 and B15 amplicons, respectively), and $5 \mu \mathrm{L}$ DNA solution. When sense or antisense amplicon strands were intended for DMA, appropriate primers (forward or reverse, respectively) were used in excess for asymmetric PCR. The reaction mixture was covered with 2 drops of mineral oil. PCR conditions were as follows: initial denaturation for $5 \mathrm{~min}$ at $95^{\circ} \mathrm{C}$, then $80-100$ cycles of $13 \mathrm{~s}$ at $95^{\circ} \mathrm{C}$ and $2 \mathrm{~s}$ at $54^{\circ} \mathrm{C}$ or $56^{\circ} \mathrm{C}$ (for N3 and B15 amplicons, respectively) with fluorescence acquisition at the annealing temperature. The fluorescence acquisition was done for the signal(s) of the TaqMan probes for either one or both strands. After PCR, the DNA was heated at $95^{\circ} \mathrm{C}$ for $1 \mathrm{~min}$, cooled and incubated at $55^{\circ} \mathrm{C}$ for $2 \mathrm{~min}$, after which it was melted from $55^{\circ} \mathrm{C}$ to $85^{\circ} \mathrm{C}$ (increments of $0.4^{\circ} \mathrm{C}$, dwell time of $6 \mathrm{~s}$, rate of heating of $3.3^{\circ} \mathrm{C} / \mathrm{s}$ ).

The presence of both sense and antisense TaqMan probes in the reaction mixture allows, on the one hand, monitoring of real-time PCR by hydrolysis of each probe, and on the other, efficient blocking of wild-type allele amplification. It should be noted that only one of the two TaqMan probes (sense or antisense, designated by subscripts) takes part in the DMA, namely the one that is complementary to the excess amplicon strand.

All experiments were carried out on the Bio-Rad CFX96 platform using optical channels with excitation/detection wavelengths of 560-590/610-650 nm and 620-650/675-690 nm for the ROX and Cy5 fluorophores, respectively. The Bio-Rad CFX96 Manager software (version

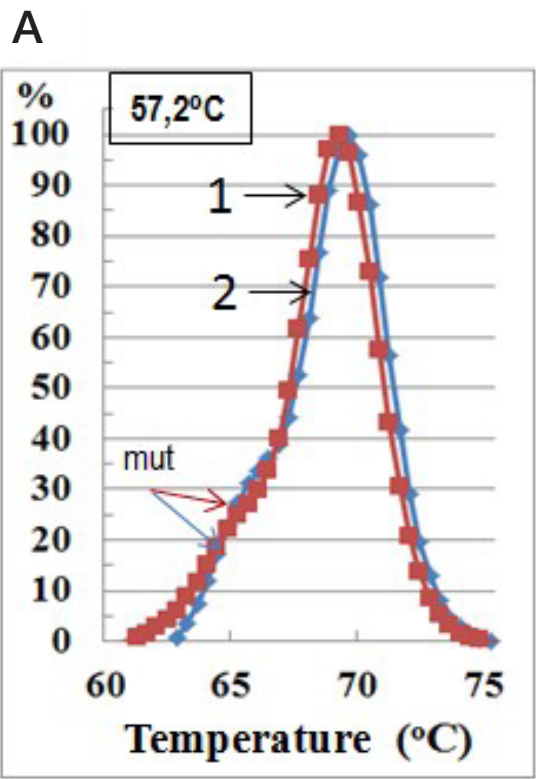

B
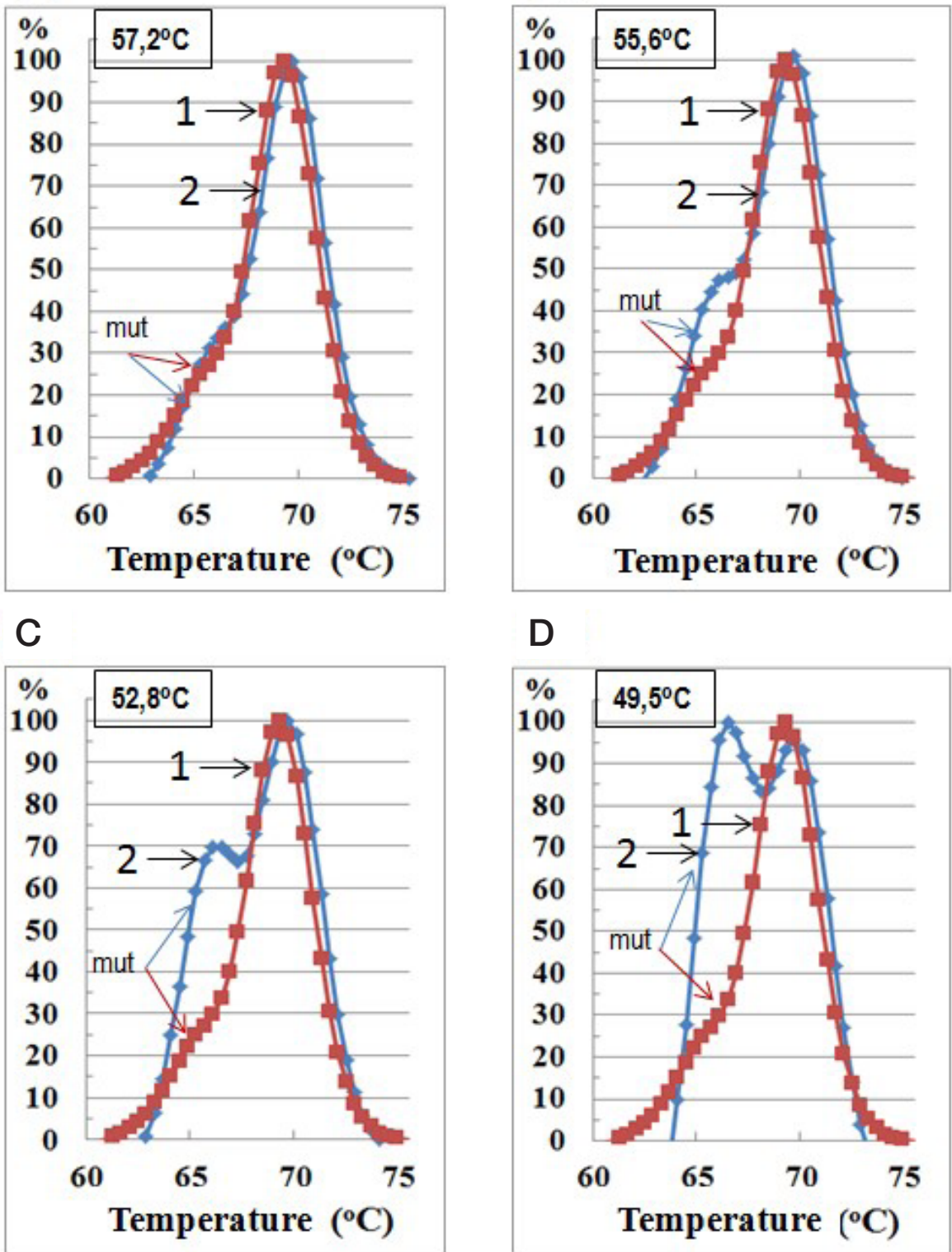

D

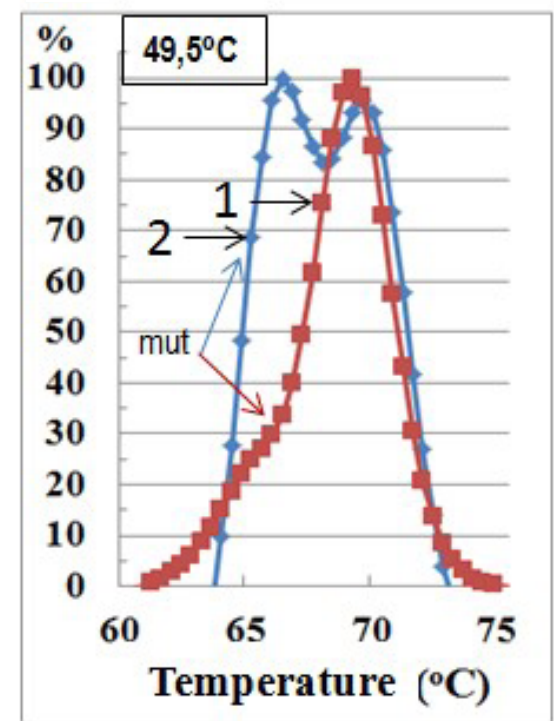

Figure 2. Effect of annealing temperature on amplification enrichment of a mutant allele. Asymmetric PCR with a gradient of annealing temperature was followed by DNA melting analysis (DMA). A DNA sample containing 5\% mutant BRAF (V600E) was assayed under standard conditions [Curve 1: B15-ROX(30) TaqMan probe absent during PCR was added at the DMA step] and modified conditions [Curve 2: both B15-ROX(30) and B15-Cy5(26) TaqMan probes were present during PCR and DMA, but only B15-ROX(30) , probe hybridized with excess antisense strand manifested itself in the DNA melting analysis]. The data were exported to Microsoft Excel, and the melt peaks were normalized relative to the maximum value taken as $100 \%$. The annealing temperatures are indicated in each Panel $(A-D)$. The arrows indicate positions of the heteroduplexes reflecting the mutant alleles.

1.6) was used to collect and analyze amplification and melting data. In some cases, fluorescence data (the negative derivative of fluorescence versus temperature) were exported to Microsoft Excel for normalization to the highest peak height.

\section{Results and discussion}

The possibility of using TaqMan probes not only to monitor real-time PCR, for which they were originally intended (16), but also for DMA (13) substantially simplifies and reduces the cost of this method, increases its efficiency, and expands its possible uses, in particular for mutation scanning of the KRAS, NRAS, BRAF and PIK3CA genes $(14,15)$.

Here, asymmetric PCR is used for the preferential amplification of one of the two strands of the amplicon. When this strand is hybridized with the TaqMan probe, the 
Panel A

(a)

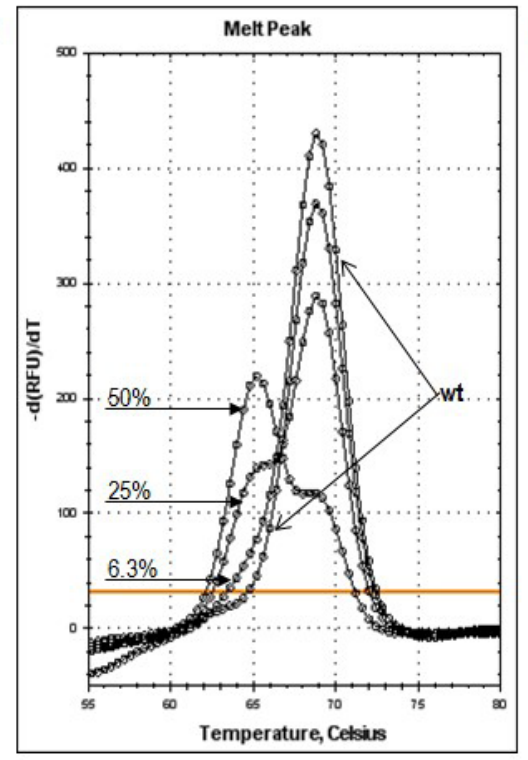

(b)

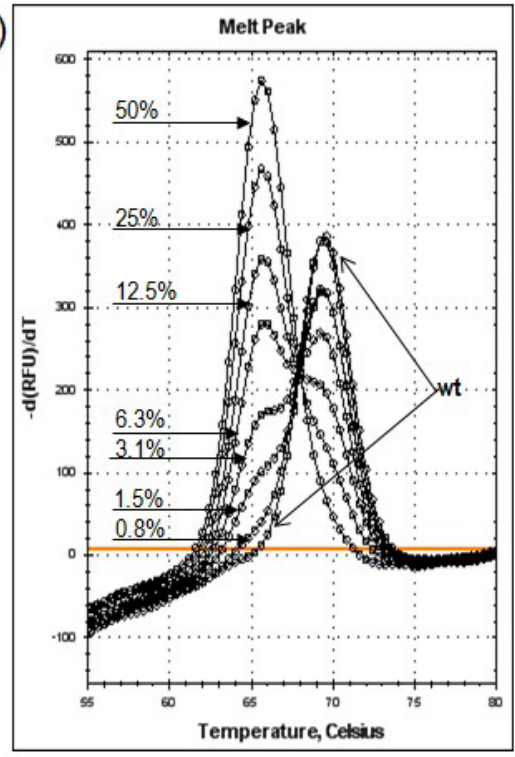

Panel B

(a)

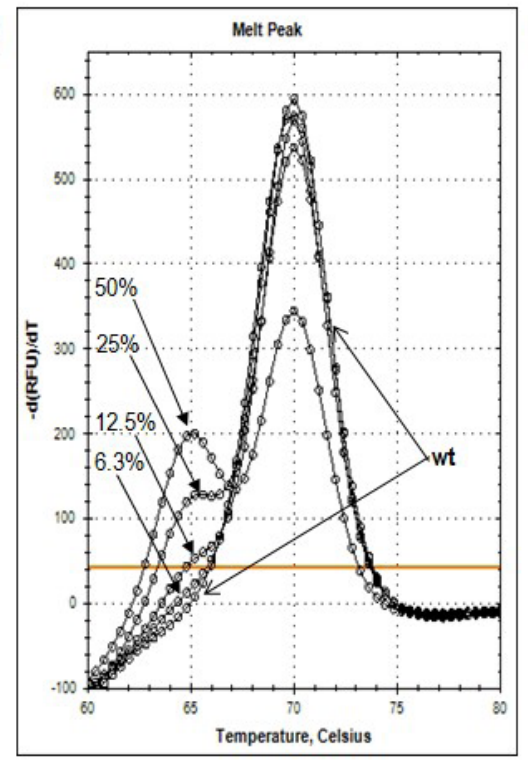

(b)

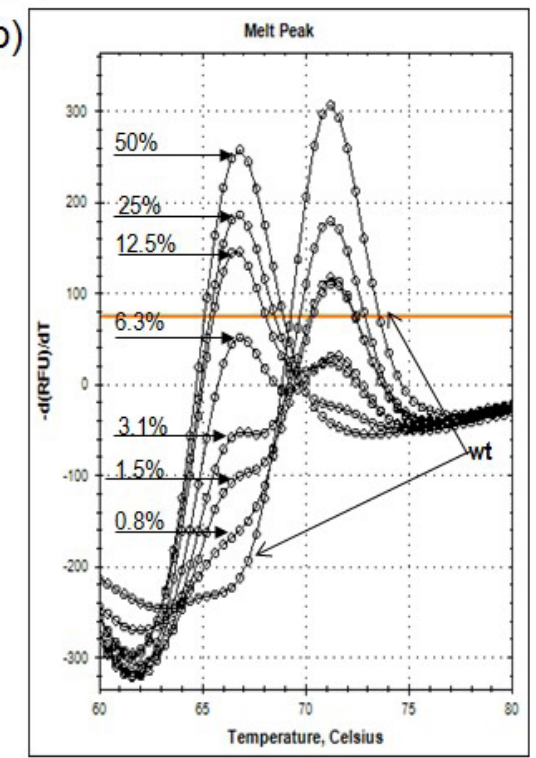

Figure 3. Sensitivity testing of DNA melting analysis (DMA) under standard (DMA $\left.A_{s t}\right)$ and modified (DMA ${ }_{\text {mod }}$ ) conditions. Sensitivity testing of mutant BRAF (GTG600GAG) (Panel $\mathrm{A}$ ) and NRAS (CAA61AAA) (Panel B) genes by DMA $\mathrm{s}_{\mathrm{st}}$ and $\mathrm{DMA}_{\text {mod }}$ was carried out using serial dilutions of mutant DNA samples with wild-type DNA. Subpanels (a) and (b) represent $\mathrm{DMA}_{\mathrm{st}}$ and $\mathrm{DMA}_{\text {mod }}$, respectively. The melt peaks of the wild-type and mutant alleles are denoted by arrows showing the percentage of mutant DNA. In the case of DMA $A_{\mathrm{s}}$, the TaqMan probes [B15-ROX(30) and N3-ROX(30), for BRAF and NRAS amplicons, respectively] were added after amplification was completed just prior to DMA. In the case of DMA $A_{\text {mod }}$, both sense and antisense TaqMan probes were present in the PCR and DMA, but only the sense TaqMan probes hybridized with excess antisense strands manifested themselves in melting.

fluorophore and the quencher in the probe become spatially separated, enhancing the fluorescence of the probe. After thermal denaturation of the duplex, the probe is released and becomes folded in a random coil, and consequently, the fluorescence becomes quenched. The TaqMan probe/ amplicon strand duplex melting curve permits discrimination of the wild-type and mutant alleles. It is noteworthy that melt peaks of the alternative duplexes (sense probe/antisense strand and antisense probe/sense strand) differ significantly by both the $T_{m}$ and $\Delta T_{m}$ values (the latter is the difference between the $T_{m}$ values of the homo- and heteroduplex). The better resolution of the melt peaks improves DMA sensitivity and facilitates discrimination between the mutant and wild-type alleles (14). Therefore, the bi-strand DMA may increase the reliability of mutation scanning (Supplementary Figure S1).

There were some data to suggest that TaqMan probes might be able to block wild-type allele amplification and subsequently increase the sensitivity of mutation detection. First, we have noticed that in the presence of $1 \mu \mathrm{M}$ TaqMan probe in the reaction mixture of asymmetric PCR with EvaGreen, the $\mathrm{C}_{\mathrm{q}}$ (quantification cycle) value became increased by 1.0-1.5 (in the case of a symmetric reaction no such increase has been noticed) (Supplementary Figure S2). Second, it was shown recently that the extension rate of Taq polymerase was significantly reduced when some secondary structures are designed into the template region or an oligonucleotide probe is present (17). These findings suggest that the Taq polymerase slows down when it has to remove an obstacle in its way using its $5^{\prime}-3^{\prime}$ exonuclease activity. Therefore, it seemed possible to preferentially amplify the mutant allele since the heteroduplex with the mismatch is less stable (and, hence, less obstructive for the enzyme) than the perfect homoduplex; the thermodynamic melting point software (18) predicts a decrease in $\mathrm{T}_{\mathrm{m}}$ of $3^{\circ} \mathrm{C}-7^{\circ} \mathrm{C}$ per mismatch.

Presumably, a relatively small difference in stability (and, hence, in blocking activity) of perfect and mismatched probe/template duplexes can manifest itself during amplification only under suboptimal PCR conditions. Keeping this in mind, we carried out preliminary experiments to test: (i) short amplification cycles ( $2 \mathrm{~s}$ for the annealing and elongation steps instead of $20 \mathrm{~s}$ ); (ii) the presence of an intercalating dye; (iii) high concentrations of both TaqMan probes; and (iv) low enzyme concentrations (in serial dilutions of Taq polymerase made to determine the optimal concentration). Each of these factors incrementally increased the enrichment of the mutant allele (Supplementary Figure S3), and the maximum effect was achieved by adjusting the annealing temperature. The number of cycles of the asymmetric PCR was increased to 80-100.

In designing the TaqMan probes for DMA, it was necessary to make the optimal trade-off between the strength of the fluorescence signal (the longer the 
probe, the stronger the signal due to a longer distance between the fluorophore and quencher), the resolution of the melt peaks of wild-type and mutant alleles (the shorter the probe, the better the resolution due to a stronger destabilizing effect of a mismatch), and the blocking activity of the probe (which increases with the probe length). The optimal lengths of the TaqMan probes appeared to be between 24 and 30 bases.

There were two reasons to use two TaqMan probes for PCR-DMA analysis. First, it appeared that the two probes together are more effective in blocking wild-type amplification than either one alone. Second, using two TaqMan probes instead of one TaqMan probe plus a normal oligonucleotide with 3 '-end blocking, provides the possibility of testing both amplicon strands for a mutation. It is difficult to foresee which of these tests would be more sensitive for a specific mutation (see Supplementary Figure S1), so the analyses of complementary strands can supplement each other (14).

To test the TaqMan probes as blocking agents, we used a DNA sample containing $5 \%$ of the most common BRAF V600E (T1799A) mutation, made by mixing DNA from normal and RKO cells. Figure 2 presents the results of enriched amplification of the mutant BRAF allele by asymmetric PCR with the TaqMan probes at various annealing temperatures (Curves 1 and 2 correspond to standard and modified conditions of PCR, respectively). It can be seen that as the annealing temperature is decreased (Figure 2, A-D) and the duplexes become more stable and obstructive, the enrichment of the mutant allele becomes more pronounced (the mutant melt peak grows from hardly discernible to dominant). The maximum enrichment of the mutant allele is achieved at an annealing temperature of $\sim 50^{\circ} \mathrm{C}$ (lower temperatures completely block the amplification). It should be noted that in subsequent experiments we had to adjust the annealing temperature since the gradient PCR conditions allow the observation of the allele enrichment phenomenon but do not guarantee the accuracy of determining the annealing temperature.

Figure 3 presents sensitivity testing of mutant BRAF (V600E) and NRAS (Q61K) genes by both the DMA ${ }_{\text {st }}$ and DMA ${ }_{\text {mod }}$ procedures. It can be seen that $6.3 \%$ (a)

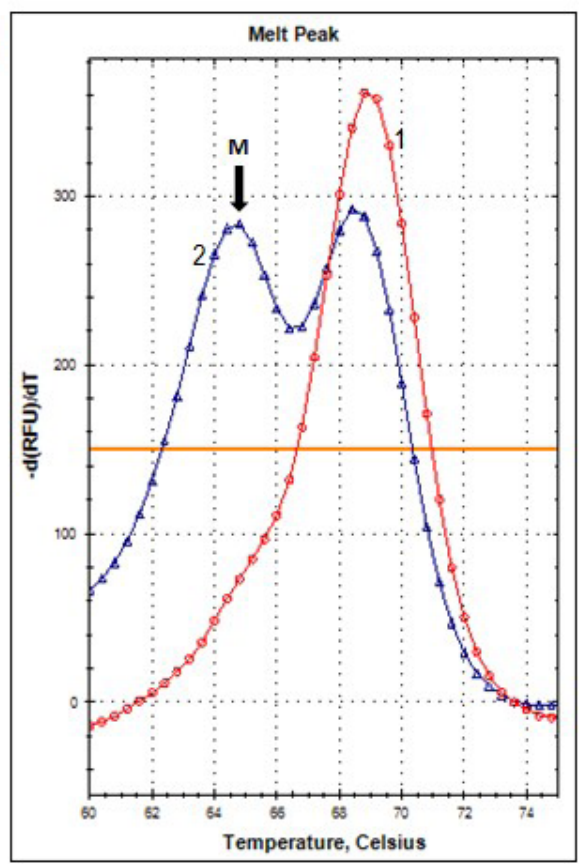

Panel A

(b)

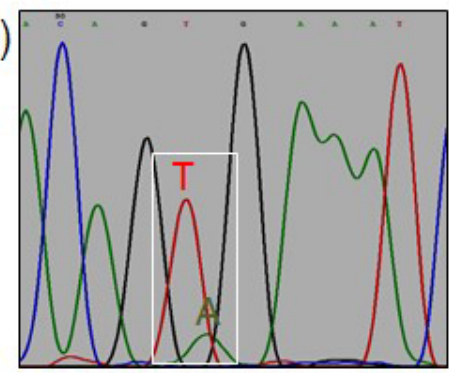

(c)

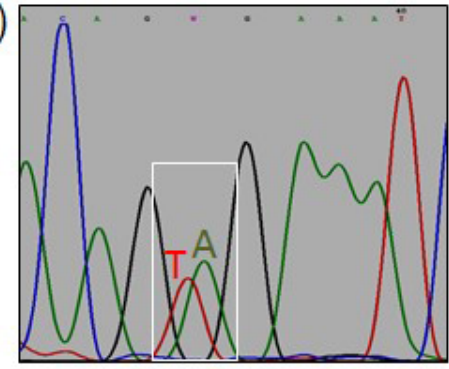

Panel B

(a)

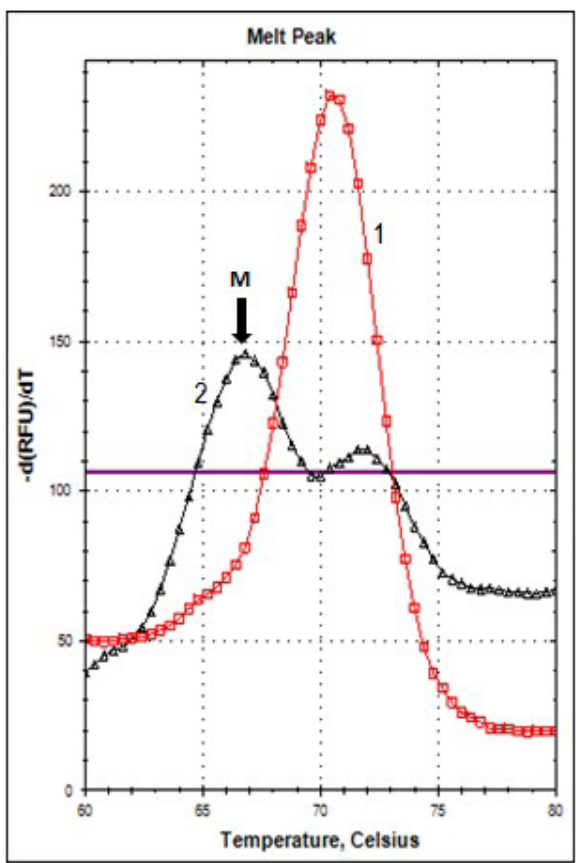

(b)

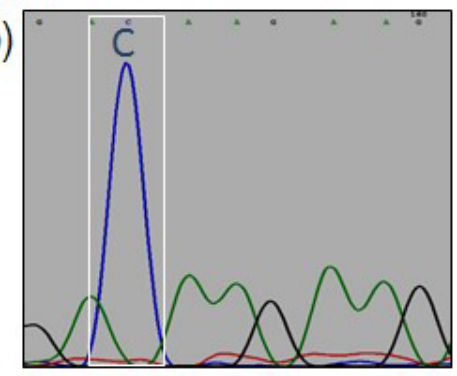

(c)

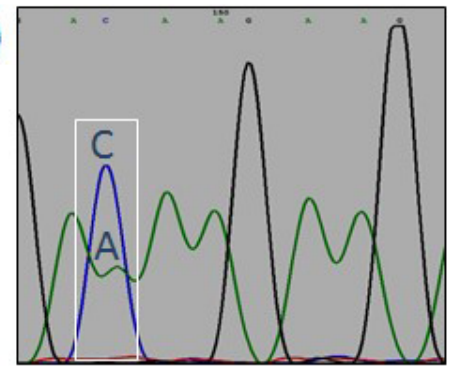

Figure 4. Verification of DNA melting analysis (DMA) results for standard $\left(D M A_{s t}\right)$ and modified $\left(D M A_{\text {mod }}\right)$ conditions by Sanger sequencing. Panel $A$ : DNA sample containing $5 \%$ mutant BRAF (GTG600GAG) was assayed with $\mathrm{DMA}_{\mathrm{st}}$ and $\mathrm{DMA}_{\bmod }$ (Subpanel a, Curves 1 and 2, respectively). The mutant (M) position is marked by the arrow. The amplicons produced with $\mathrm{DMA}_{\mathrm{st}}$ and $\mathrm{DMA}_{\bmod }$ were sequenced by the Sanger method (Subpanels $\mathrm{b}$ and $\mathrm{c}$, respectively); the box shows the TA replacement. Panel B: DNA sample with $3 \%$ mutant NRAS (CAA61AAA) was assayed with DMA st $_{\text {t }}$ and $\mathrm{DMA}_{\bmod }$ (Subpanel a, Curves 1 and 2, respectively). Designations are as in Panel A. The box shows the CA replacement.

and $0.8 \%$ mutant alleles are detected by these procedures, respectively, which is a significant (approximately 10-fold) increase in the sensitivity of mutation scanning. Evidently, the different nucleotide sequences of various genetic hot spots necessitate individual adjustment of enriched amplification conditions. 
Taq polymerase possesses 5'-3'-exonuclease activity (required for hydrolysis of the TaqMan probes) but lacks $3^{\prime}-5^{\prime}$-exonuclease editing activity and is relatively error-prone $\left(1-5 \times 10^{-5}\right.$ errors per base pair per doubling) $(19,20)$. Therefore, the amplicons may carry dispersed nucleotide substitutions whose number grows during PCR. One can hypothesize that the increased cycling (up to 80-100 cycles) of asymmetric PCR used for DMA mod $_{\text {can }}$ introduce a pseudo-mutation in the probe binding region and lead to false-positive results. This suggestion, however, seems unlikely since the amplification efficiency of asymmetric PCR is much lower than that of symmetric PCR (44\% and 93\%, respectively) and, judging from PCR end point evaluations, in most cases 100 cycles of the former carried out under suboptimal modified conditions are quantitatively equivalent to approximately 40 cycles of the latter (Supplementary Figure S4). A relatively insignificant impact of the increased cycling number on the validity of our enriched mutation scanning method has been verified by Sanger sequencing. We assayed DNA samples with 5\% of mutant BRAF (GTG600GAG, panel A) and $3 \%$ of mutant NRAS (CAA61AAA, panel B) by $D M A_{s t}$ and $D M A_{\text {mod }}$, after which the PCR products were sequenced (Figure 4). Dispersed pseudo-mutations caused by Taq polymerase errors, if any, could lead to a slightly elevated background level but do not obscure the prominent mutation signal. Thus, only enriched PCR combining asymmetric PCR with TaqMan probes allowed us to unequivocally reveal the mutations by both methods (DMA and Sanger sequencing), whereas the standard PCR failed to do so in either case. Similar analyses of DNA samples isolated from formalin-fixed paraffin-embedded tissues and containing BRAF and NRAS mutations (GTG600GAG, Panel A, and CAA61CTA, Panel $B$, respectively) are presented in Supplementary Figure S5.

Compared with the commonly employed blocking agents (peptide nucleic acids) for enrichment of the mutant alleles (21), our proposed approach has the combined benefits of high flexibility, simplicity, and cost-efficiency since the same dual-labeled oligonucleotide is used for three independent functions: monitoring real-time PCR, enriching amplification of the mutant alleles, and DNA melting analysis. This eliminates the need for the costly chemical synthesis of peptide nucleic acids. These properties make our proposed approach useful for both clinical diagnostics and the discovery of mutation hot spots in the human genome.

\section{Author contributions}

I.V.B. and A.V.L. designed the study. A.M.S. and A.I.S. prepared DNA samples. I.V.B., I.O.P., A.M.S., A.I.S., V.N.K., and V.P.S. performed the experiments and critically revised the data. A.V.L. wrote the manuscript.

\section{Acknowledgments}

This work was done with support from the State Federal Budget Program N 62.2.3.

\section{Competing interests}

The authors declare no competing interests.

\section{References}

1. Vogelstein, B., N. Papadopoulos, V.E. Velculescu, S. Zhou, L.A. Diaz, and K.W. Kinzler. 2013. Cancer genome landscapes. Science 339:1546-1558

2. Alexandrov, L.B., S. Nik-Zainal, D.C. Wedge, S.A. Aparicio, S. Behjati, A.V. Biankin, G.R. Bignell, N. Bolli, et al. 2013. Signatures of mutational processes in human cancer. Nature 500:415-421.

3. Lawrence, M.S., P. Stojanov, C.H. Mermel, J.T. Robinson, L.A. Garraway, T.R. Golub, M. Meyerson, S.B. Gabriel, et al. 2014. Discovery and saturation analysis of cancer genes across 21 tumour types. Nature 505:495-501.

4. Borràs, E., I. Jurado, I. Hernan, M.J. Gamundi, M. Dias, I. Marti, B. Mane, A. Arcusa, et al. 2011. Clinical pharmacogenomic testing of KRAS, BRAF and EGFR mutations by high resolution melting analysis and ultra-deep pyrosequencing. BMC Cancer 11:406.

5. Diaz, L.A., Jr., R.T. Williams, J. Wu, I. Kinde, J.R. Hecht, J. Berlin, B. Allen, I. Bozic, et al. 2012. The molecular evolution of acquired resistance to targeted EGFR blockade in colorectal cancers. Nature 486:537-540.

6. Misale, S., R. Yaeger, S. Hobor, E. Scala, M. Janakiraman, D. Liska, E. Valtorta, R. Schiavo, et al. 2012. Emergence of KRAS mutations and acquired resistance to anti-EGFR therapy in colorectal cancer. Nature 486:532-536.

7. Weidlich, S., K. Walsh, D. Crowther, M.E. Burczynski, G. Feuerstein, F.A. Carey, R.J.C. Steele, C.R. Wolf, et al. 2011. Pyrosequencingbased methods reveal marked inter-individual differences in oncogene mutation burden in human colorectal tumours. Br. J. Cancer 105:246-254.

8. Tsiatis, A.C., A. Norris-Kirby, R.G. Rich, M.J. Hafez, C.D. Gocke, J.R. Eshleman, and K.M. Murphy. 2010. Comparison of Sanger sequencing, pyrosequencing, and melting curve analysis for the detection of KRAS mutations: diagnostic and clinical implications. J. Mol. Diagn. 12:425-432.
9. Zhou, L., Y. Wang, and C.T. Wittwer. 2011. Rare allele enrichment and detection by allelespecific PCR, competitive probe blocking, and melting analysis. BioTechniques 50:311-318.

10. Vossen, R.H., E. Aten, A. Roos, and J.T. den Dunnen. 2009. High-resolution melting analysis (HRMA): more than just sequence variant screening. Hum. Mutat. 30:860-866.

11. Vorkas, P.A., N. Poumpouridou, S. Agelaki, C. Kroupis, V. Georgoulias, and E.S. Lianidou. 2010. PIK3CA hotspot mutation scanning by a novel and highly sensitive highresolution small amplicon melting analysis method. J. Mol. Diagn. 12:697-704.

12. Erali, M. and C.T. Wittwer. 2010. High resolution melting analysis for gene scanning. Methods 50:250-261.

13. Huang, Q., Z. Liu, Y. Liao, X. Chen, Y. Zhang, and Q. Li. 2011. Multiplex fluorescence melting curve analysis for mutation detection with duallabeled, self-quenched probes. PLoS One 6:e19206.

14. Botezatu, I.V., I.O. Nechaeva, A. Stroganova, A.I. Senderovich, V.N. Kondratova, V.P. Shelepov, and A.V. Lichtenstein. 2015. Optimization of melting analysis with Taqman probes for detection of KRAS, NRAS and BRAF mutations. Anal. Biochem. 491:75-83

15. Botezatu, I.V., I.O. Nechaeva, A.M. Stroganova, A.I. Senderovich, V.N. Kondratova, V.P. Shelepov, and A.V. Lichtenstein. 2015. Asymmetric real-time PCR and multiplex melting curve analysis with TaqMan probes for detecting PIK3CA mutations. Data Brief 5:913-917.

16. Livak, K.J., S.J. Flood, J. Marmaro, W. Giusti, and K. Deetz. 1995. Oligonucleotides with fluorescent dyes at opposite ends provide a quenched probe system useful for detecting PCR product and nucleic acid hybridization. PCR Methods Appl. 4:357-362.

17. Montgomery, J.L., N. Rejali, and C.T. Wittwer. 2014. The influence of nucleotide sequence and temperature on the activity of thermostable DNA polymerases. J. Mol. Diagn. 16:305-313.

18. Schütz, E. and N. von Ahsen. 1999. Spreadsheet software for thermodynamic melting point prediction of oligonucleotide hybridization with and without mismatches. BioTechniques 27:1218-1222, 1224.

19. Smith, J. and P. Modrich. 1997. Removal of polymerase-produced mutant sequences from PCR products. Proc. Natl. Acad. Sci. USA 94:6847-6850.

20. McInerney, P., P. Adams, and M.Z. Hadi. 2014. Error rate comparison during polymerase chain reaction by DNA polymerase. Mol. Biol. Int. 2014:287430.

21. Guha, M., E. Castellanos-Rizaldos, and G.M. Makrigiorgos. 2013. DISSECT method using PNA-LNA clamp improves detection of T790m mutation. PLoS One 8:e67782.

Received 05 November 2016; accepted 04 January 2017.

Address correspondence to Anatoly $V$. Lichtenstein, N.N. Blokhin Cancer Research Center, Kashirskoe Shosse 24, 115478 Moscow, Russia. E-mail: alicht@mail.ru

To purchase reprints of this article, contact: biotechniques@fosterprinting.com 